\title{
Article
}

\section{An Evaluation of a Simplified Impression Membrane Sampling Method for the Diagnosis of Microbial Keratitis}

\author{
Tobi F. Somerville ${ }^{1,2, *}$, Rose Herbert ${ }^{1,2}\left(\mathbb{D}\right.$, Timothy Neal ${ }^{3}$, Malcolm Horsburgh ${ }^{4}$ and Stephen B. Kaye ${ }^{1,2}(\mathbb{D}$ \\ 1 Department of Eye and Vision Sciences, University of Liverpool, Liverpool L69 3BX, UK; \\ Rose.Herber!@liverpoolft.nhs.uk (R.H.); sbkaye@liverpool.ac.uk (S.B.K.) \\ 2 St Paul's Eye Unit, Liverpool University Hospitals NHS Foundation Trust, Liverpool L7 8XP, UK \\ 3 Department of Microbiology, Liverpool University Hospitals NHS Foundation Trust, Liverpool L9 3GA, UK; \\ Timothy.Neal@liverpoolft.nhs.uk \\ 4 Department of Infection Biology and Microbiomes, University of Liverpool, Liverpool L69 3BX, UK; \\ malhorsb@liverpool.ac.uk \\ * Correspondence: tobi@liverpool.ac.uk
}

check for updates

Citation: Somerville, T.F.; Herbert,

R.; Neal, T.; Horsburgh, M.; Kaye, S.B.

An Evaluation of a Simplified

Impression Membrane Sampling

Method for the Diagnosis of

Microbial Keratitis. J. Clin. Med. 2021,

10, 5671. https://doi.org/10.3390/

jcm10235671

Academic Editors: Francesco Aiello and Marco Ciotti

Received: 14 November 2021

Accepted: 29 November 2021

Published: 30 November 2021

Publisher's Note: MDPI stays neutral with regard to jurisdictional claims in published maps and institutional affiliations.

Copyright: (c) 2021 by the authors. Licensee MDPI, Basel, Switzerland. This article is an open access article distributed under the terms and conditions of the Creative Commons Attribution (CC BY) license (https:/ / creativecommons.org/licenses/by/ $4.0 /)$.

\begin{abstract}
The purpose of this study was to compare bacterial isolation rate using a corneal impression membrane (CIM) and a sharp instrument for obtaining corneal samples from patients with suspected microbial keratitis (MK). Data was retrospectively collected for all patients that had corneal samples taken for presumed MK between May 2014 and May 2020. Prior to May 2017 samples were collected by scraping the edges of the ulcer with a blade. From May 2017, samples were collected by placing a CIM (Millicell cell culture insert) against the ulcer. All corneal samples were processed using the same conventional diagnostic culture method. A total of 3099 corneal samples were included, of which 1214 (39.2\%) were corneal scrapes and 1885 (60.9\%) CIMs. Microorganisms were isolated from 235 (19.4\%) and 1229 (65.2\%) cases using a corneal scrape and CIM, respectively $(p<0.001)$. Of routinely described pathogenic microorganisms, there were significant increases in the isolations of S. aureus (2.4\% to $11.3 \%$ ) and Serratia ( $0.5 \%$ to $1.7 \%)$ using the CIM and no significant changes in the isolations of $S$. pneumoniae and P. aeruginosa. No significant differences were seen between the isolation rates of fungi or Acanthamoeba species. There was a significant increase in the isolation rates of other Streptococcal species ( $0.7 \%$ to $6.9 \%)$ and CNS species, specifically, S. epidermidis $(2.1 \%$ to $26.2 \%)$, S. capitis ( $0.4 \%$ to $2.6 \%$ ) and S. warneri $(0.3 \%$ to $1.6 \%)$ using the CIM. The simplified CIM sampling method is an effective method for collecting corneal samples from patients with presumed MK in clinical practice.
\end{abstract}

Keywords: microbial keratitis; corneal scrape; corneal impression membrane

\section{Introduction}

Microbial keratitis (MK) is an ophthalmological emergency that can lead to sight threatening complications such as corneal scarring, perforation, endophthalmitis and ultimately blindness [1-4]. Improving outcomes depends on accurate and rapid identification of the causative microorganism.

The need to detect bacteria, fungi, yeasts and protozoa together with the fact that there may be relatively few microorganisms in a corneal ulcer, means that an adequate sample must be obtained and cultured on a variety of different media [5]. This has led to the traditional practice of taking multiple samples by scraping the edges of the ulcer with a blade, needle or spatula and directly plating the material onto several culture media. The likely causative microorganism is often only isolated in $50 \%$ of cases using traditional scraping methods and standard conventional diagnostic culture (CDC) [6]. This, in addition to good activity of fourth generation fluoroquinolones, may explain the reluctance of some ophthalmologists to perform a corneal scrape to reach a microbiological diagnosis [7,8]. It 
is evident that improvements are required to both facilitate and simplify the collection of samples and the detection and diagnosis of microorganisms in suspected MK.

In 2015, we developed a minimally invasive sampling method using an impression membrane made from polytetrafluroethylene (PTFE) [9]. In 130 clinically suspected MK patients, samples were collected in a random order using a traditional corneal scraping method and a $4 \mathrm{~mm}$ cut-out sterile PTFE membrane that was placed onto the ulcer using sterile forceps for $5 \mathrm{~s}$ before being placed into enrichment culture broth and transported to the laboratory for conventional diagnostic culture. The isolation of microorganisms from cases of clinically suspected MK was found to be significantly higher using the cut-out PTFE membrane compared to corneal scrapes, $40.8 \%$ versus $26.9 \%$, respectively [9]. We have reported that use of the PTFE membrane also improves the detection rate of HSV-1 [10] and have demonstrated that microorganisms remain stable on the PTFE membrane for prolonged storage periods [11].

In practice, however, a $4 \mathrm{~mm}$ punched-out PTFE membrane requires the use of fine sterile forceps to place the membrane onto the surface of the eye, meaning that specialist ophthalmic skills and a slit-lamp biomicroscope are required to undertake the sampling. The use of a $12 \mathrm{~mm}$ cell culture insert containing the same membrane material overcomes these practicality issues and, as such, was implemented into routine clinical practice for obtaining corneal samples in cases of suspected MK in our department in May 2017. The aim of this study was to compare the microbial isolation rate using a $12 \mathrm{~mm}$ PTFE Millicell cell culture insert and a sharp instrument for obtaining corneal samples from patients with suspected MK in routine practice.

\section{Materials and Methods}

Data was collected from the Department of Microbiology at the Liverpool University Hospitals NHS Foundation Trust for all patients who had corneal samples taken for clinically suspected MK between May 2014 and May 2020.

Samples were collected from ulcers prior to May 2017 by scraping the edges of the ulcer with a number 11 blade (Swann Morton, Sheffield, UK) whilst wearing sterile gloves, as previously described [9]. The blade was placed into $0.5 \mathrm{mLs}$ of enrichment culture broth, (BHI) and transported to the laboratory at room temperature. From May 2017, samples were collected from ulcers by placing the $12 \mathrm{~mm}$ hydrophilic PTFE membrane of a Millicell cell culture insert (CIM, corneal impression membrane) over the ulcer for 2-3 s whilst wearing sterile gloves (Figure 1). The eyelids were held apart and care was taken to avoid contact with the eyelids or conjunctiva. The CIM was placed into $9 \mathrm{mLs}$ of BHI enrichment broth and transported to the laboratory. Conventional diagnostic culture was performed as previously described [9].
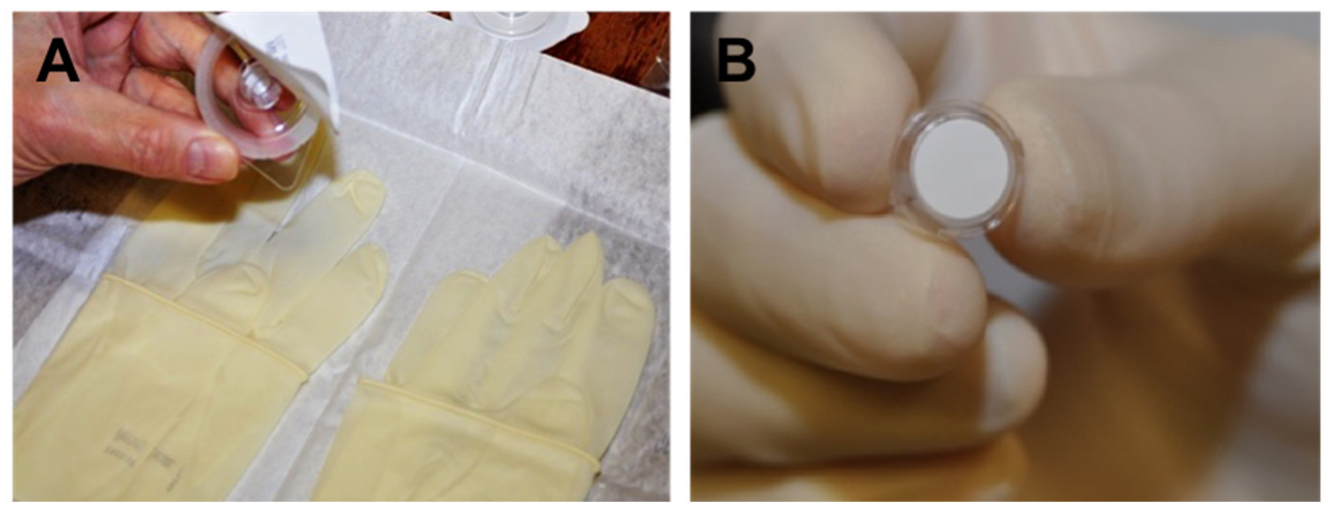

Figure 1. Displays the sterile pre-packaged Millicell cell culture insert being tipped onto sterile field (A) and the Millicell cell culture insert being held in the sampler's dominant hand prior to sample collection (B). 
Microorganisms were classified as those routinely described as pathogenic in MK (S. aureus, S. pneumoniae, Pseudomonas aeruginosa and Serratia, Candida, Fusarium, Aspergillus and Acanthamoeba species) and those of unknown significance [12].

All data collected in this study were entered into an electronic database via Microsoft Excel 2018 and analysed using SPSS (version 27). The $\chi^{2}$ was used as indicated for the analysis of categorical variables. When there were fewer than five observations in any cell, Fisher's exact test was used. Post hoc analysis was carried out using a Bonferroni correction for multiple comparisons.

\section{Results}

Over the 6-year study period, a total of 3099 corneal samples were included. The average age of patients was 49.6 years (SD 18.8). A total of 368 patients had more than one corneal sample taken during the study period, either during the same or a recurrent keratitis episode. There was a significant increase in the number of corneal samples obtained in the 3-year period following the implementation of the CIM into clinical practice compared to the 3-year period prior to its implementation, despite numbers of ophthalmic emergency attendances remaining stable (Table 1). Overall, 1214 (39.2\%) of the samples were obtained using a corneal scrape and $1885(60.9 \%)$ using a CIM, respectively $(p<0.001)$ (Table 1$)$. No adverse events associated with the CIM were recorded during the study period.

Table 1. Number of corneal samples obtained in the 3-year periods pre- and post the implementation of corneal impression membrane (CIM) sampling into our clinical practice compared to the number of ophthalmic emergency attendances to our unit in the same time period.

\begin{tabular}{ccc}
\hline & $\begin{array}{c}\text { Corneal Scrapes } \\
\text { (May 2014-April 2017) }\end{array}$ & $\begin{array}{c}\text { CIM } \\
\text { (May 2017-April 2020) }\end{array}$ \\
\hline $\begin{array}{c}\text { Number of corneal samples collected } \\
\text { Number of ophthalmic emergency attendances to } \\
\text { our department }\end{array}$ & 1214 & 1885 \\
Corneal samples as a \% of ophthalmic emergencies & 51,617 & 51,281 \\
\hline
\end{tabular}

CIM: corneal impression membrane.

Microorganisms were isolated from 235 (19.4\%) cases using a corneal scrape and $1229(65.2 \%)$ using a CIM $(p<0.001)$ (Table 2$)$. Significantly higher isolation rates of both routinely described pathogenic microorganisms and microorganisms of unknown significance in MK were seen in the CIM group (Table 2). More than one isolate was identified in $30(2.5 \%)$ and $272(14.4 \%)$ of positive samples using a corneal scrape and CIM, respectively (Table 2).

Table 2. Isolates from cases of clinically suspected microbial keratitis over a 6-year period.

\begin{tabular}{cccc}
\hline Isolated Organism & $\begin{array}{c}\text { Corneal Scrapes } \\
(\boldsymbol{n}=\mathbf{1 2 1 4})\end{array}$ & $\begin{array}{c}\text { CIM } \\
(\boldsymbol{n}=\mathbf{1 8 8 5})\end{array}$ & $\boldsymbol{p}$-Value \\
\hline $\begin{array}{c}\text { Positive samples } \\
\text { Routinely described pathogenic } \\
\text { microorganisms in MK }\end{array}$ & $235(19.4 \%)$ & $1229(65.2 \%)$ & $<0.001$ \\
$\begin{array}{c}\text { Microorganisms of unknown } \\
\text { significance in MK }\end{array}$ & $87(7.2 \%)$ & $334(17.7 \%)$ & $<0.001$ \\
Mixed samples & $152(12.5 \%)$ & $950(50.4 \%)$ & $<0.001$ \\
\hline
\end{tabular}

CIM: corneal impression membrane; MK: microbial keratitis.

\subsection{Isolated Microorganisms}

Of accepted MK pathogenic bacteria (12), there was a significant increase in the isolation of S. aureus $(p<0.001)$ and Serratia spp. $(p=0.048)$ using the CIM sampling method and no significant changes in the isolation of: S. pneumoniae and P. aeruginosa. No significant differences were seen between the isolation rates of Candida, Fusarium and Aspergillus species or Acanthamoeba species (Table 3). 
Table 3. Routinely described pathogenic microorganisms identified from cases of clinically suspected microbial keratitis over a 6-year period.

\begin{tabular}{cccc}
\hline Isolated Organism & $\begin{array}{c}\text { Corneal Scrapes } \\
(\boldsymbol{n}=\mathbf{1 2 1 4})\end{array}$ & $\begin{array}{c}\text { CIM } \\
(\boldsymbol{n}=\mathbf{1 8 8 5})\end{array}$ & $p$-Value \\
\hline $\begin{array}{c}\text { Gram-positive } \\
\text { bacteria }\end{array}$ & & & \\
$\begin{array}{c}\text { S. aureus } \\
\text { S. pneumoniae }\end{array}$ & $29(2.4 \%)$ & $213(11.3 \%)$ & $<0.001$ \\
Gram-negative & $13(1.1 \%)$ & $33(1.8 \%)$ & 1.0 \\
$\quad$ bacteria & & & 1.0 \\
$\begin{array}{c}\text { P. aeruginosa } \\
\text { Serratia spp. }\end{array}$ & $30(2.5 \%)$ & $60(3.2 \%)$ & 0.048 \\
$\quad$ Fungi & $6(0.5 \%)$ & $32(1.7 \%)$ & 1.0 \\
Candida spp. & $3(0.2 \%)$ & $4(0.2 \%)$ & 1.0 \\
Fusarium spp. & $1(0.08 \%)$ & 0 & 1.0 \\
Aspergillus spp. & $4(0.3 \%)$ & $2(0.1 \%)$ & 1.0 \\
Protozoa & $4(0.3 \%)$ & $3(0.2 \%)$ & \\
Acanthamoeba spp. & & &
\end{tabular}

CIM: corneal impression membrane.

Of those microorganisms of unknown clinical significance in MK, there was a significant increase in the isolation of both Streptococcal species other than S. pneumoniae and CNS species, specifically S. epidermidis, S. capitis and S. warneri (Table 4 and Table S1, Supplementary Data). No significant differences were seen between the isolation rates of Gram-negative bacteria and fungal species of unknown clinical significance in MK.

Table 4. Microorganisms of unknown significance identified from cases of clinically suspected microbial keratitis over a 6-year period.

\begin{tabular}{cccc}
\hline Isolated Organism & $\begin{array}{c}\text { Corneal Scrapes } \\
(\boldsymbol{n}=\mathbf{1 2 1 4})\end{array}$ & $\begin{array}{c}\text { CIM } \\
(\boldsymbol{n}=\mathbf{1 8 8 5})\end{array}$ & $p$-Value \\
\hline $\begin{array}{c}\text { Gram-positive } \\
\text { bacteria }\end{array}$ & $116(9.6 \%)$ & $921(48.9 \%)$ & $<0.001$ \\
$\begin{array}{c}\text { Other streptococci } \\
\text { species }\end{array}$ & $9(0.7 \%)$ & $130(6.9 \%)$ & $<0.001$ \\
$\begin{array}{c}\text { CNS } \\
\text { Others }\end{array}$ & $76(6.3 \%)$ & $799(42.4 \%)$ & $<0.001$ \\
$\begin{array}{c}\text { Gram-negative } \\
\text { bacteria }^{\text {b }}\end{array}$ & $37(3.0 \%)$ & $61(3.2 \%)$ & 1.0 \\
Fungi $^{c}$ & $44(3.6 \%)$ & $96(5.1 \%)$ & 0.30 \\
\hline
\end{tabular}

CNS: Coagulase-negative Staphylococcus. ${ }^{*}$ CNS species are shown in Table S1 in the Supplementary Data. ${ }^{\text {a }}$ Other Gram-positive bacteria include: Enterococcus spp., Kocuria spp., Micrococcus spp., Rothia spp., Aerococcus spp., Corynebacterium spp., Diphtheroid spp., and Bacillus spp. b Gram-negative bacteria include: Moraxella spp., E. Coli, other unclassified Enterobacteriaceae spp., Morganella spp., Brevundimonas sp., Chryseobacterium sp. Citrobacter spp., Delftia sp., Acinetobacter spp., Haemophilus spp., Klebsiella spp., Pantoea spp., Proteus spp., Raoultella spp., Stenotrophomonas spp., Achromobacter spp. and Alcaligenes sp. ' Fungi include: Pencillium spp., Acremonium spp., Phoma sp., Scedospirium sp., Ulocladium sp. and Yeasts.

\subsection{Polymicrobial Keratitis}

Significantly more corneal samples in the CIM group were found to have more than one microorganism isolated, $2.4 \%$ versus $14.4 \%$, respectively $(p<0.001)$ (Table 2$)$. There was no significant difference in the numbers and distribution of microorganisms in each mixed sample between the corneal scrape and CIM sampling groups (Table S2, Supplementary Data). Gram-positive bacteria were the most frequent microorganism group isolated in the mixed samples in both sampling groups with CNS being the most frequently isolated organism group in all of the mixed samples. Gram-positive polybacterial infection, in which two or more species of Gram-positive bacteria were isolated, was the most common microbial growth pattern in both sampling methods. One CIM sample had mixed infection with five species of microorganism: E. coli, Haemophilus haemolyticus, Streptococcus mitis, 
an alpha-haemolytic Streptococci (AHS) and a Yeast sp. Of the mixed bacterial and fungal samples, all three corneal scrapes had mixed growth with Gram-positive bacteria, nine $(3.3 \%)$ of the CIMs had mixed growth with Gram-positive bacteria and four $(1.5 \%)$ had mixed growth with Gram-negative bacteria. There was only one mixed Acanthamoeba infection identified which had mixed growth with CNS.

\section{Discussion}

Identification of the causative microorganism is crucial for successful treatment and improving outcomes in MK. Culture of the causative microorganism remains the gold standard procedure and is necessary to determine the susceptibility profile of the pathogen. In this study, we demonstrated that the introduction of a simplified CIM sampling method into clinical practice was associated with a significant increase in the number of corneal samples collected and in microorganism isolation rates. We demonstrated the simplified $\mathrm{CIM}$ to be equivalent or better at isolating routinely described pathogenic microorganisms in MK compared to obtaining a corneal scrape. The inclusion of a large number of corneal samples obtained by multiple ophthalmic practitioners from a large range of patients with a wide spectrum of disease suggest the results will be applicable to corneal sampling in everyday ophthalmic clinical practice.

In the past, there have been several attempts to simplify corneal sample collection. Indirect plating methods that involve placing the sampling device into transport medium prior to plating on to agar plates reduces the need to collect multiple scrape samples and has been shown to be equivalent to directly plating corneal scrape samples in the clinic in terms of microorganism isolation rates [13-15]. We found that the isolation rate using the CIM (65.2\%) was significantly higher than when we used a surgical blade and indirect culture, and is also higher or equivalent to those reported for other absorbent sampling devices including the direct culture of cotton tipped swabs (43.9\%) [16], Mini-tip Culturettes (41.7\%) [17], the indirect culture of nylon-tipped swabs (69\%) [18] and the $4 \mathrm{~mm}$ PTFE membrane that we have previously tested (40.8\%) [9]. In addition, the CIM has the benefit of being non-invasive and simple to localize to the corneal ulcer.

The spectrum of microorganisms identified in both sampling groups was similar, with Gram-positive bacteria the most frequent group isolated, and is comparable to many other studies conducted in the UK [19-22]. The CIM, in addition to demonstrating comparatively equivalent isolation rates of routinely described MK pathogenic microorganisms: S. pneumoniae, P. aeruginosa, Candida sp., Aspergillus sp. Fusarium sp. And Acanthamoeba, also demonstrated significant increases in the isolation rates of S. aureus and Serratia bacteria. This has important treatment implications as $S$. aureus is known to be the most frequently associated microorganism in recurrent MK and Gram-negative bacteria such as Serratia are associated with a more rapid inflammatory destructive course and worse visual outcomes [23-26].

The Biopore membrane in the cell culture insert is made from PTFE with pore size 0.4. PTFE of similar pore size was demonstrated to have the largest cellular yield when tested against other material types and the large $12 \mathrm{~mm}$ diameter size of the membrane in the Millicell cell culture insert increases the chances of covering the entire corneal ulcer [27]. Both of these properties could explain the increased microorganism isolation rates demonstrated in this study.

In addition to routinely described pathogenic microorganisms in MK, the CIM group demonstrated higher isolation rates of CNS and Streptococci species other than S. pneumoniae of which their pathogenic role in MK is unclear. These isolation rates were also higher than those reported using a similar one-touch method with a swab and transport medium [18]. Isolation rates of these microorganisms could be a result of several hypothesised theories. Firstly, for reasons previously discussed, the CIM sampling method is better at picking up microorganisms from the corneal ulcer itself compared to corneal scraping methods and, therefore, these microorganisms may play a larger role in the pathogenesis of MK than previously thought. The importance of identifying CNS to the species level is becoming 
more recognised as virulence factors such as exopolysaccharide, and lipase and protease enzymatic production are increasingly identified as potential virulence factors in MK development [28]. Secondly, the large surface area of the Millicell cell culture insert may favour the uptake of transient commensals that may be present on the patient's lids, conjunctiva, tear film or 'unaffected' corneal epithelium. It is not known whether a 'normal corneal microbiome' exists as previous corneal sampling methodologies have been invasive and, therefore, have been able to sample unaffected and healthy corneas. Next-generation sequencing of the conjunctiva has demonstrated a diverse ocular surface microbiome made up of Corynebacterium, Acinetobacter, Pseudomonas, Staphylococcus, Cutibacterium and Streptococcus [29]. It is, therefore, possible that movement of the tear film across the ocular surface increases the yield of identified microorganisms during the CIM sampling process.

Polymicrobial keratitis has been previously estimated to have a $2-8 \%$ incidence rate in the literature and is associated with larger corneal infiltrate size and greater mean duration for resolution of infection [30,31]. The higher rates of polymicrobial infection seen in the CIM sampling group could similarly be a result of a better microorganism pick up rate from the ulcer itself by the CIM or represent increased inadvertent sampling of commensals as discussed. The fact that the distribution of microorganisms was not seen to differ significantly between the corneal sampling methods, however, would suggest this is not due to increased commensal organism detection.

We hypothesize that the increased numbers of corneal samples obtained in our unit post implementation of the CIM despite ophthalmic attendances remaining stable is likely to be secondary to the comparatively easier sampling methodology of the CIM for both the ophthalmic practitioner and the MK patient. The Millicell cell culture inserts are presterilised and individually packed by the manufacturer making them easy to implement in a clinical setting. The cell cultures plastic mount means that the culture insert can easily be held in the hand of the user and placed over the ulcer safely without the need for sharp instruments such as forceps. Furthermore, the technique does not require the use of a slit-lamp biomicroscope, meaning that corneal samples can be obtained by allied health care professionals and in settings where access to slit-lamp biomicroscopes may be limited or not practical. The CIM requires much less patient cooperation than traditional corneal scraping methods and in the authors experience, is much better tolerated by the typical MK patient.

Although the study had a large sample size and was set over a long study duration, it is limited by its retrospective design. It is also comparing the sampling methods over two consecutive time periods and, therefore, it is unknown whether microbe detection could have been influenced by other variables that fluctuate over time such as seasonal and temperature variations [32], changing contact lens habits and hygiene [33], systemic comorbidity rates [34] and incidence and prevalence of other MK risk factors such as steroid eye drop use and ocular surface conditions [35]. UK studies looking at MK isolates over the time period used in this study are lacking. Results from five UK case series that observed MK isolates prior to this study period have conflicting results [19-22,36]. Furthermore, confounding effects of previous antimicrobial use and corneal ulcer characteristics on the isolation rates of each sampling group cannot be ruled out as the corneal scrape and CIM samples were not taken from the same corneal ulcer.

In conclusion, we demonstrated that the introduction of a simplified CIM sampling method into routine ophthalmic clinical practice was associated with a significant increase in the number of corneal samples collected and an increase in the culture positivity rate. The CIM was found to be better at isolating pathogenic microorganisms in MK and compared to traditional scraping methods, is easier to perform and requires much less patient corporation. A CIM is a safe and effective method for collecting corneal samples from patients with presumed MK in routine ophthalmic practice and is associated with higher isolation rates compared to corneal scrapes. 
Supplementary Materials: The following are available online at https: / www.mdpi.com/article/ 10.3390/jcm10235671/s1: Table S1: Coagulase-negative staphylococcus isolates, Table S2: Corneal samples with mixed microorganism growth.

Author Contributions: Conceptualization, T.F.S., T.N. and S.B.K.; methodology, T.F.S., T.N. and S.B.K.; data curation: T.F.S.; formal analysis, T.F.S. and R.H.; writing-original draft preparation, T.F.S.; writing-review and editing, T.F.S., R.H., T.N., M.H., S.B.K. All authors have read and agreed to the published version of the manuscript.

Funding: This research received no external funding. Tobi F Somerville is the recipient of a Medical Research Council Clinical Research Training Fellowship.

Institutional Review Board Statement: The CIM was reviewed by the Liverpool University Hospitals NHS Foundation Trust internal device board who approved its use for collection of corneal samples from patients with suspected microbial keratitis in routine clinical practice. This was an audit of routinely collected clinical data that was locally approved by the Liverpool University Hospitals NHS Foundation Trust and, therefore, ethical approval was not required.

Informed Consent Statement: The data from this study was generated from an audit of routine clinical practice.

Data Availability Statement: The data presented in this study are available on request from the corresponding author.

Conflicts of Interest: The authors declare no conflict of interest.

\section{References}

1. Ibrahim, Y.W.; Boase, D.L.; Cree, I.A. Epidemiological characteristics, predisposing factors and microbiological profiles of infectious corneal ulcers: The Portsmouth corneal ulcer study. Br. J. Ophthalmol. 2009, 93, 1319-1324. [CrossRef] [PubMed]

2. Henry, C.R.; Flynn, H.W., Jr.; Miller, D.; Forster, R.K.; Alfonso, E.C. Infectious keratitis progressing to endophthalmitis: A 15-year study of microbiology, associated factors, and clinical outcomes. Ophthalmology 2012, 119, 2443-2449. [CrossRef] [PubMed]

3. Jin, H.; Parker, W.T.; Law, N.W.; Clarke, C.L.; Gisseman, J.D.; Pflugfelder, S.C.; Al-Mohtaseb, Z.N. Evolving risk factors and antibiotic sensitivity patterns for microbial keratitis at a large county hospital. Br. J. Ophthalmol. 2017, 101, 1483-1487. [CrossRef]

4. Ting, D.S.J.; Cairns, J.; Gopal, B.P.; Ho, C.S.; Krstic, L.; Elsahn, A.; Dua, H.S. Risk Factors, Clinical Outcomes, and Prognostic Factors of Bacterial Keratitis: The Nottingham Infectious Keratitis Study. Front. Med. 2021, 8, 715118. [CrossRef]

5. Moshirfar, M.; Hopping, G.C.; Vaidyanathan, U.; Liu, H.; Somani, A.N.; Ronquillo, Y.C.; Hoopes, P.C. Biological Staining and Culturing in Infectious Keratitis: Controversy in Clinical Utility. Med. Hypothesis Discov. Innov. Ophthalmol. J. $2019,8,145-151$.

6. Ung, L.; Bispo, P.J.M.; Shanbhag, S.S.; Gilmore, M.S.; Chodosh, J. The persistent dilemma of microbial keratitis: Global burden, diagnosis, and antimicrobial resistance. Surv. Ophthalmol. 2019, 64, 255-271. [CrossRef] [PubMed]

7. McDonnell, P.J. Empirical or Culture-Guided Therapy for Microbial Keratitis? Arch. Ophthalmol. 1996, 114, 84-87. [CrossRef]

8. Vajpayee, R.B.; Dada, T.; Saxena, R.; Vajpayee, M.; Taylor, H.R.; Venkatesh, P.; Sharma, N. Study of the first contact management profile of cases of infectious keratitis: A hospital-based study. Cornea 2000, 19, 52-56. [CrossRef]

9. Kaye, S.; Sueke, H.; Romano, V.; Chen, J.Y.; Carnt, N.; Tuft, S.; Neal, T. Impression membrane for the diagnosis of microbial keratitis. Br. J. Ophthalmol. 2016, 100, 607-610. [CrossRef]

10. Brunner, M.; Somerville, T.; Corless, C.E.; Myneni, J.; Rajhbeharrysingh, T.; Tiew, S.; Kaye, S.B. Use of a corneal impression membrane and PCR for the detection of herpes simplex virus type-1. J. Med. Microbiol. 2019, 68, 1324-1329. [CrossRef]

11. Somerville, T.F.; Corless, C.E.; Neal, T.; Kaye, S.B. Effect of storage time and temperature on the detection of Pseudomonas aeruginosa, Acanthamoeba and Herpes Simplex Virus from corneal impression membranes. J. Med. Microbiol. 2018, 67, 1321-1325. [CrossRef]

12. Lakhundi, S.; Siddiqui, R.; Khan, N.A. Pathogenesis of microbial keratitis. Microb. Pathog. 2017, 104, 97-109. [CrossRef]

13. Bhadang, Y.; Sharma, S.; Das, S.; Sahu, S. Role of liquid culture media in the laboratory diagnosis of microbial keratitis. Am. J. Ophthalmol. 2013, 156, 745-751. [CrossRef] [PubMed]

14. Kaye, S.B.; Rao, P.G.; Smith, G.; Scott, J.A.; Hoyles, S.; Morton, C.E.; Harvey, G. Simplifying collection of corneal specimens in cases of suspected bacterial keratitis. J. Clin. Microbiol. 2003, 41, 3192-3197. [CrossRef]

15. Kratz, A.; Levy, J.; Klemperer, I.; Lifshitz, T. Broth cultures yield vs traditional approach in the workup of infectious keratitis. Eye 2006, 20, 215-220. [CrossRef] [PubMed]

16. Sagerfors, S.; Ejdervik-Lindblad, B.; Söderquist, B. Does the sampling instrument influence corneal culture outcome in patients with infectious keratitis? A retrospective study comparing cotton tipped applicator with knife blade. BMJ Open Ophthalmol. 2020, 5, e000363. Available online: http:/ /bmjophth.bmj.com/content/5/1/e000363.abstract (accessed on 11 January 2021). [CrossRef]

17. Epley, K.D.; Katz, H.R.; Herling, I.; Lasky, J.B. Platinum spatula versus Mini-tip Culturette in culturing bacterial keratitis. Cornea 1998, 17, 74-78. Available online: http://europepmc.org/abstract/MED/9436884 (accessed on 11 January 2011). [CrossRef] 
18. Pakzad-Vaezi, K.; Levasseur, S.D.; Schendel, S.; Mark, S.; Mathias, R.; Roscoe, D.; Holland, S.P. The Corneal Ulcer One-Touch Study: A Simplified Microbiological Specimen Collection Method. Am. J. Ophthalmol. 2015, 159, 37-43. [CrossRef]

19. Tavassoli, S.; Nayar, G.; Darcy, K.; Grzeda, M.; Luck, J.; Williams, O.M.; Tole, D. An 11-year analysis of microbial keratitis in the South West of England using brain-heart infusion broth. Eye 2019, 33, 1619-1625. [CrossRef] [PubMed]

20. Tan, S.Z.; Walkden, A.; Au, L.; Fullwood, C.; Hamilton, A.; Qamruddin, A.; Carley, F. Twelve-year analysis of microbial keratitis trends at a UK tertiary hospital. Eye 2017, 31, 1229-1236. [CrossRef]

21. Orlans, H.O.; Hornby, S.J.; Bowler, I.C.J.W. In vitro antibiotic susceptibility patterns of bacterial keratitis isolates in Oxford, UK: A 10-year review. Eye 2011, 25, 489-493. [CrossRef]

22. Ting, D.S.J.; Settle, C.; Morgan, S.J.; Baylis, O.; Ghosh, S. A 10-year analysis of microbiological profiles of microbial keratitis: The North East England Study. Eye 2018, 32, 1416-1417. [CrossRef] [PubMed]

23. Bourcier, T.; Thomas, F.; Borderie, V.; Chaumeil, C.; Laroche, L. Bacterial keratitis: Predisposing factors, clinical and 249 microbiological review of 300 cases. Br. J. Ophthalmol. 2003, 87, 834. Available online: http://bjo.bmj.com/content/87/7/834 .abstract (accessed on 1 November 2021). [CrossRef] [PubMed]

24. Okonkwo, A.C.O.; Siah, W.F.; Hogg, H.D.J.; Anwar, H.; Figueiredo, F.C. Microbial keratitis in corneal grafts: Predisposing factors and outcomes. Eye 2018, 32, 775-781. [CrossRef] [PubMed]

25. Kaye, R.; Kaye, A.; Sueke, H.; Neal, T.; Winstanley, C.; Horsburgh, M.; Kaye, S. Recurrent Bacterial Keratitis. Investig. Opthalmol. Vis. Sci. 2013, 54, 4136-4139. [CrossRef]

26. Somerville, T.F.; Shankar, J.; Aldwinckle, S.; Sueke, H.; Neal, T.; Horsburgh, M.J.; Kaye, S.B. Recurrent microbial keratitis and endogenous site Staphylococcus aureus colonisation. Sci. Rep. 2020, 10, 1-6.

27. Mokhtarzadeh, M.; Casey, R.; Glasgow, B.J. Flourescein punctate staining traced to superficial corneal epithelial cells by impression cytology and confocal microscopy. Investig. Ophthalmol. Vis. Sci. 2011, 52, 2127-2135. [CrossRef] [PubMed]

28. Priya, R.; Mythili, A.; Singh, Y.R.B.; Sreekumar, H.; Manikandan, P.; Panneerselvam, K.; Shobana, C.S. Virulence, Speciation and Antibiotic Susceptibility of Ocular Coagualase Negative Staphylococci (CoNS). J. Clin. Diagn. Res. 2014, 8, DC33-DC37.

29. Delbeke, H.; Younas, S.; Casteels, I.; Joossens, M. Current knowledge on the human eye microbiome: A systematic review of available amplicon and metagenomic sequencing data. Acta Ophthalmol. 2021, 99, 16-25. [CrossRef]

30. Jones, D.B. Polymicrobial keratitis. Transations Am. Ophthalmol. Soc. 1981, 79, 153-167.

31. Lim, N.C.S.; Lim, D.K.A.; Ray, M. Polymicrobial Versus Monomicrobial Keratitis. Eye Contact Lens Sci. Clin. Pract. 2013, 39, 348-354. [CrossRef] [PubMed]

32. Walkden, A.; Fullwood, C.; Tan, S.; Au, L.; Armstrong, M.; Brahma, A.K.; Carley, F. Association Between Season, Temperature and Causative Organism in Microbial Keratitis in the UK. Cornea 2018, 37, 1555-1560. [CrossRef]

33. Morgan, P.B.; Efron, N.; Helland, M.; Itoi, M.; Jones, D.; Nichols, J.J.; Woods, C.A. Global trends in prescribing contact lenses for extended wear. Contact Lens Anterior Eye 2011, 34, 32-35. [CrossRef] [PubMed]

34. Kingston, A.; Robinson, L.; Booth, H.; Knapp, M.; Jagger, C.; MODEM Project. Projections of multi-morbidity in the older population in England to 2035: Estimates from the Population Ageing and Care Simulation (PACSim) model. Age Ageing 2018, 47, 374-380. [CrossRef] [PubMed]

35. Dana, R.; Bradley, J.L.; Guerin, A.; Pivneva, I.; Stillman, I.O.; Evans, A.M.; Schaumberg, D.A. Estimated prevalence and incidence of dry eye disease based on coding analysis of a large, all-age united States health care system. Am. J. Ophthalmol. 2019, 202, 47-54. [CrossRef] [PubMed]

36. Shalchi, Z.; Gurbaxani, A.; Baker, M.; Nash, J. Antibiotic resistance in microbial keratitis: Ten-year experience of corneal scrapes in the United Kingdom. Ophthalmology 2011, 118, 2161-2165. [CrossRef] 